\title{
ON GENERALIZATION OF CONTINUED FRACTION OF GAUSS
}

\author{
REMY Y. DENIS
}

Department of Mathematics

University of Gorakhpur

Gorakhpur - 273009

INDIA

(Received May 10, 1988)

ABSTRACT. In this paper we establish a continued fraction represetation for the ratio af two basic bilateral hypergeometric series $2^{\psi} 2^{\prime}$ shich generalize Gauss' continued fraction for the ratio of two $2 F_{1}$ 's.

KEY WORDS AND PHRASES. Continued fractions and hypergeometric series. 1980 AMS SUBJECT CLASSIFICATION CODE. 11 A55.

1. INTRODUCTION.

Gauss (see Wall [3] and also Jones and Thron [2], gave the following continued fraction involving the ratio of two Gaussian ${ }_{2} F_{1}$ 's,

$$
\begin{aligned}
& F\left[\begin{array}{c}
a, b+1 ; z \\
c+1
\end{array}\right] / F\left[\begin{array}{c}
a, b ; z \\
c
\end{array}\right] \\
& =\frac{1}{1-\frac{a(c-b) z / c(c+1)}{\frac{(b+1)(c-a+1) z /(c+1)(c+2)}{(a+1)(c-b+1) z /(c+2)(c+3)}}} \\
& 1-\frac{(b+2)(c-a+2) z /(c+3)(c+4)}{1-\frac{(b-a)}{1-}}
\end{aligned}
$$

where

$$
2 F_{1}\left[\begin{array}{c}
\alpha, \beta ; z \\
\gamma
\end{array}\right]=\sum_{n=0}^{\infty} \frac{[\alpha]_{n}[\beta]_{n} z^{n}}{[1]]_{n}[\gamma]_{n}},(|z|<1)
$$

in which the symbol $[\alpha]_{n}$ stands for $\alpha(\alpha+1)(\alpha+2) \ldots(\alpha+n-1)$ and $[\alpha]_{0}=1$.

In this paper we establish the continued fraction for the ratio

$$
2^{\psi} 2\left[\begin{array}{l}
\alpha, \beta ; x \\
\delta, \gamma
\end{array}\right] / 22^{\psi} 2\left[\begin{array}{l}
\alpha, \beta q ; x \\
\delta, \gamma q
\end{array}\right]
$$

where

$$
2 \psi_{2}\left[\begin{array}{l}
\alpha, \beta ; x \\
\delta, \gamma
\end{array}\right]=\sum_{n=-\infty}^{\infty} \frac{[\alpha]_{n}[\beta]_{n} x^{n}}{[\delta]_{n}[\gamma]_{n}},(|\delta \gamma / \alpha \beta|<|x|<1,|q|<1),
$$


where

$$
[\alpha]_{n} \equiv[\alpha ; q]_{n}=(1-\alpha)(1-\alpha q) \ldots\left(1-\alpha q{ }^{n-1}\right),[\alpha]_{0}=1
$$

The other notations appearing in this paper carry their usual meaning.

2. MAIN RESULT.

In this paper we establish the following result

$$
\begin{aligned}
& { }_{2} \psi_{2}\left[\begin{array}{l}
\alpha, \beta q ; x \\
\delta, \gamma q
\end{array}\right] / 2 \psi_{2}\left[\begin{array}{l}
\alpha, \beta ; x \\
\delta, \gamma
\end{array}\right] \\
& =\frac{1}{A_{0}}+\frac{x{ }_{0}}{C_{0}}+\frac{x D_{0}}{A_{1}}+\frac{x B_{1}}{C_{1}}+\frac{x D_{1}}{A_{2}}+\frac{x B_{2}}{C_{2}}+\cdots \cdot
\end{aligned}
$$

where for $1=0,1,2,3, \ldots$

$$
\begin{aligned}
& A_{i}=\frac{\left(1-\beta q^{i}\right)\left(\gamma q^{2 i+1}-\delta\right)}{\left(1-\gamma q^{2 i}\right)\left(B q^{i+1}-\delta\right)}, \\
& B_{i}=\frac{q^{i+1}\left(1-\alpha q^{i}\right)\left(1-\beta q^{i}\right)\left(\beta-\gamma q^{i}\right)}{\left(1-\gamma q^{2 i+1}\right)\left(1-\gamma q^{2 i}\right)\left(B q^{i+1}-\delta\right)} \text {, } \\
& c_{1}=\frac{\left(1-\alpha q^{i}\right)\left(\gamma q^{2 i+2}-\delta\right)}{\left(1-\gamma q^{2 i+1}\right)\left(\alpha q^{i+1}-\delta\right)}
\end{aligned}
$$

and

$$
D_{i}=\frac{q^{i+1}\left(1-\beta q^{1+1}\right)\left(1-\alpha q^{i}\right)\left(\alpha-\gamma q^{i+1}\right)}{\left(1-\gamma q^{2 i+1}\right)\left(1-\gamma q^{2 i+2}\right)\left(\alpha q^{1+1}-\delta\right)}
$$

PROOF of (2.1). It is easy to see that the following relation is true (for nonnegative integral i),

$$
\begin{aligned}
& 2^{\psi_{2}}\left[\begin{array}{cc}
\alpha q^{1}, B q^{i} ; x \\
\delta, & \gamma q^{21}
\end{array}\right]
\end{aligned}
$$

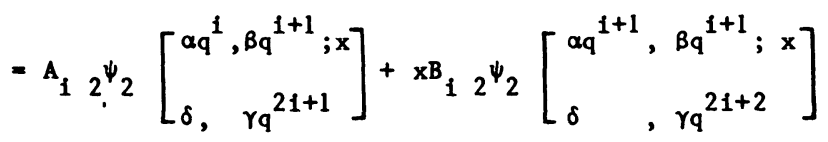

Now, interchanging $\alpha$ and $\beta$ in, (2.2) aqd then replacing $\beta$ by $B q$ and $\gamma$ by $Y q$ in it, we get

$$
\begin{aligned}
& 2^{\psi}{ }^{\psi}\left[\begin{array}{cc}
\alpha q^{1}, & B q^{1+1} ; x^{\prime} \\
\delta, & \gamma q^{2 i+1}
\end{array}\right] \\
& =C_{12} \psi_{2}\left[\begin{array}{cc}
\alpha q^{1+1}, & \beta q^{1+1} ; x \\
\delta & , \gamma q^{21+2}
\end{array}\right]+x D_{12} \psi_{2}\left[\begin{array}{cc}
\alpha q^{1+1}, \beta q^{1+2} ; x \\
\delta, \gamma q^{21+3}
\end{array}\right]
\end{aligned}
$$


Now from (2.2) for $1=0$, we get

$$
\begin{aligned}
& 2^{\psi} 2\left[\begin{array}{c}
\alpha, \beta ; x \\
\delta, \gamma
\end{array}\right] / 2^{\psi_{2}}\left[\begin{array}{c}
\alpha, \beta q ; x \\
\delta, \gamma q
\end{array}\right] \\
& =A_{0}+\frac{x_{0}}{2^{\psi_{2}}\left[\begin{array}{l}
\alpha, \beta q ; x \\
\delta, \gamma q
\end{array}\right] / 2^{\psi_{2}}\left[\begin{array}{c}
\alpha q, B q ; x \\
\delta, \gamma q{ }^{2}
\end{array}\right]}
\end{aligned}
$$

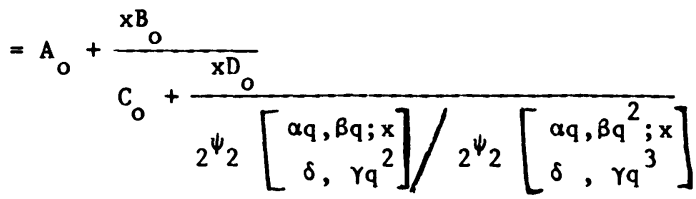

from (2.3) with $i=0$

$$
\begin{aligned}
& =A_{0}+\frac{X_{0}}{C_{0}+\frac{X D_{0}}{X B}}
\end{aligned}
$$

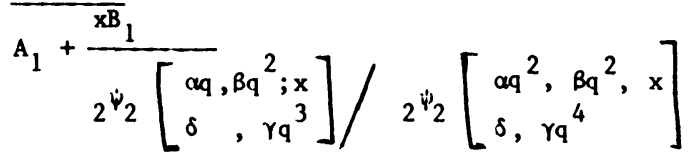

from (2.2) with $1=1$

$$
=A_{0}+\frac{x B_{0}}{C_{0}}+\frac{x D_{0}}{A_{1}}+\frac{x B_{1}}{C_{1}}+\frac{x D_{1}}{A_{2}}+\frac{x B_{2}}{C_{2}}+\cdots \cdot
$$

(by repeated application of $(2.2)$ and $(2.3)$ ). This proves $(2.1)$.

\section{SPECIAL CASES.}

Here we shall reduce certain interesting special cases of (2.1).If in (2.1) we take $\delta=q$, we get

$$
\begin{aligned}
& 2_{1}^{\Phi}\left[\begin{array}{c}
\alpha, \beta q ; x \\
\gamma q
\end{array}\right] / 22_{1}\left[\begin{array}{c}
\alpha, \beta ; x \\
\gamma
\end{array}\right] \\
& =\frac{1}{1+}+\frac{x \mu_{0}}{1}+\frac{x \nu_{0}}{1}+\frac{x \eta_{1}}{1}+\frac{x \nu_{1}}{1}+\frac{x \mu_{2}}{1}+\cdots,
\end{aligned}
$$

where for $1=0,1,2, \ldots$.

$$
\mu_{1}=q^{1}\left(1-\alpha q^{1}\right)\left(\gamma q^{i}-\beta\right) /\left(1-\gamma q^{21}\right)\left(1-\gamma q^{2 i+1}\right)
$$

and

$$
v_{i}=q^{1}\left(1-\beta q^{i+1}\right)\left(\gamma q^{1+1}-\alpha\right) /\left(1-\gamma q^{2 i+1}\right)\left(1-\gamma q^{2 i+2}\right)
$$

If $q \rightarrow 1$ in (3.1), we get (1.1), the continued fraction of Gauss.

If in (3.1) we take $B=1$ and replace $Y$ by $Y / q$, we get, 


$$
\begin{aligned}
& 2^{\$}\left[\begin{array}{cc}
\alpha, & \mathcal{q} ; x \\
\gamma
\end{array}\right] \\
& =\frac{1}{1}+\frac{x \mu_{0}}{1}+\frac{x \nu_{0}}{1}+\frac{x \mu_{1}}{1}+\frac{x \nu_{1}}{1}+\frac{x \mu_{2}}{1}+\cdots,
\end{aligned}
$$

where for $i=0,1,2, \ldots$.

and

$$
\mu_{i}=-q^{i}\left(1-\alpha q^{i}\right)\left(1-\gamma q^{i-1}\right) /\left(1-\gamma q^{2 i-1}\right)\left(1-\gamma q^{2 i}\right)
$$

$$
v_{i}=-\alpha q^{i}\left(1-q^{i+1}\right)\left(1-\gamma^{i} / \alpha\right) /\left(1-\gamma q^{2 i}\right)\left(1-\gamma q{ }^{2 i+1}\right) \text {. }
$$

Now, if in (3.2) we let $q \rightarrow 1$, we get the following known result [2]

$$
\begin{aligned}
& F\left[\begin{array}{c}
\alpha, 1 ; x \\
\gamma
\end{array}\right] \\
& =\frac{1}{1}-\frac{x \xi_{0}}{1}-\frac{x \eta_{0}}{1}-\frac{x \xi_{1}}{1}-\frac{x \eta_{1}}{1}-\frac{x \xi_{2}}{1} \ldots .
\end{aligned}
$$

where for $1=0,1,2, \ldots$.

$$
\xi_{i}=(x+1)(\gamma+1-1) /(\gamma+2 i-1)(\gamma+2 i)
$$

and

$$
n_{i}=(i+1)(\gamma-\alpha+1) /(\gamma+2 i)(\gamma+2 i+1) .
$$

If we put $\gamma=0$ in (3.2) and replace $x$ by $x q / \alpha$ and then let $\alpha \rightarrow \infty$, we get the following interesting result

$$
\begin{gathered}
\sum_{n=0}^{\infty}(-)^{n} q^{n(n+1) / 2} x^{n} \\
=\frac{1}{1}+\frac{x q}{1}+\frac{x q(q-1)}{1}+\frac{x q^{3}}{1}+\frac{x q^{2}\left(q^{2}-1\right)}{1}+\frac{2 q^{5}}{1}+\frac{x q^{3}\left(q^{3}-1\right)}{1}+\cdots,
\end{gathered}
$$

If we take $\gamma=q$ in (3.2) we get a continued fraction representation for ${ }_{1}{ }_{0}[\alpha ;-; x]$ which, when $q \rightarrow 1$, yields the continued fraction representation for general binomial $(1-x)^{-\alpha}$.

Again, if we take $\alpha=q, \gamma=q^{2}$ and replace $x$ by $-x$ in (3.2), we get a continued fraction representation for ${ }_{2} \Phi_{1}\left[q, q ; q^{2} ;-x\right]$ which, when $q \rightarrow 1$ yields the continued fraction representation for

$$
\frac{1}{x} \log (1+x)=F\left[\begin{array}{rr}
1,1 ;-x \\
2
\end{array}\right] \text {. }
$$

Similarly, we can get the continued fraction representation for

$$
\log \left(\frac{1+x}{1-x}\right)=2 x F\left[\begin{array}{c}
1 / 2,1 ; x \\
3 / 2
\end{array}\right] \text {. }
$$


Further, if we take $\alpha=0$ in (3.1), we get the following result after some simplification,

$$
\begin{aligned}
& { }^{\Phi} 1\left[\begin{array}{l}
B ; x \\
\gamma
\end{array}\right] / 1_{1}^{\infty}\left[\begin{array}{c}
\beta q_{j} x \\
\gamma q
\end{array}\right] \\
& =1+\frac{x \mu_{0}}{1}+\frac{x_{0} \nu_{0}}{1}+\frac{x \mu_{1}}{1}+\frac{x \nu_{1}}{1}+\frac{x \mu_{2}}{1}+\cdots \cdot
\end{aligned}
$$

where for $1=0,1,2, .$.

and

$$
\mu_{i}=q^{i}\left(\gamma q^{i}-\beta\right) /\left(1-\gamma q^{2 i}\right)\left(1-\gamma q^{2 i+1}\right)
$$

$$
\nu_{i}=\gamma q^{2 i+1}\left(1-B q^{i+1}\right) /\left(1-\gamma q^{2 i+1}\right)\left(1-\gamma q^{2 i+2}\right)
$$

The above (3.5) is the q-analogue of a known result [2].

Again, setting $B=1$ in (3.5) we get the continued fraction representation for $1_{1}{ }_{1}\left[\begin{array}{r}q ; x \\ q\end{array}\right]$ from which one can, for $\gamma=1$, deduce the corrsponding continued fraction expression for q-exponential function eq( $x)$ which in turn yields the continued fraction representation for exponential function $e^{2}$ when $q+1$ [2]

A number of other interesting special cases could also be deduced. The reader is referred to Wall [1] and Jones [2].

\section{ACKNOWLEDGEMENT.}

This work has been done under a Research Scheme awarded by C.S.I.R., Governinent of India, New Delhi for which the author expresses his grattude.

I am thankful to Professor R.P. Agarwal for his encouragement during the preparation of this paper.

\section{REFERENCES}

1. WALL, H.S., Analytic theory of continued fractions, D. Van Nostrand Co. Inc. New York (1948).

2. JONES, W.B. and THRON, W.J., Continued Fractions, Analytic theory and applications, Encyclopedia of Mathematics and its application, 11 Addison Wesley Publishing Company (1980).

3. ANDREWS, G.E., Ramaujan's "LOST" Notebook III. The Rogers-Ramanujan Continued Fraction. Advances in Mathematics, 41(2), (1981), 186-208. 


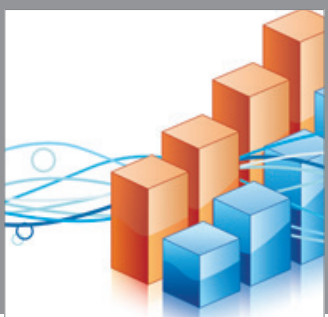

Advances in

Operations Research

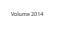

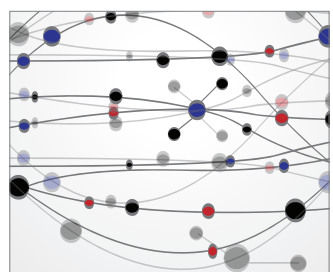

\section{The Scientific} World Journal
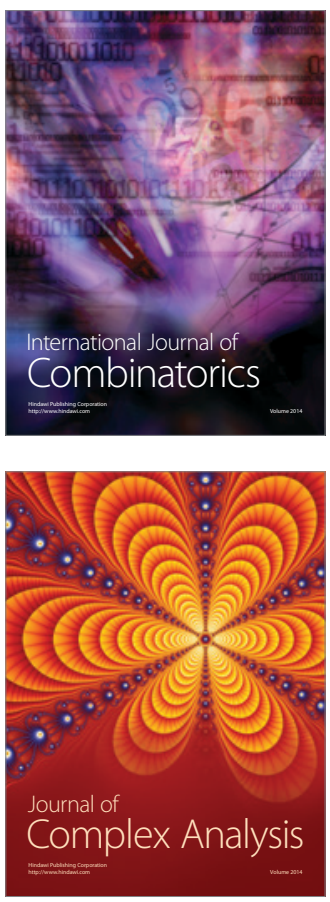

International Journal of

Mathematics and

Mathematical

Sciences
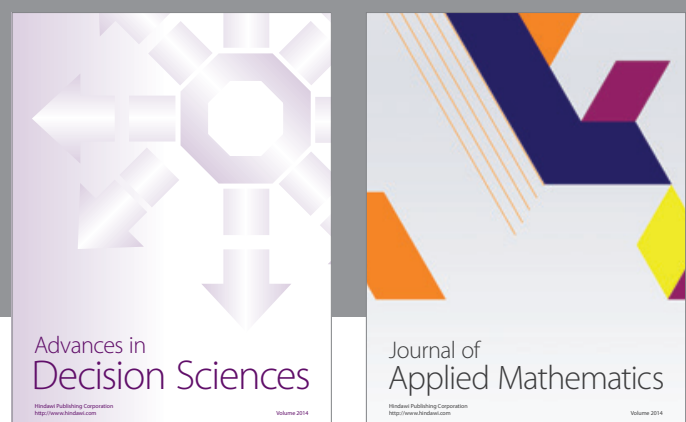

Journal of

Applied Mathematics
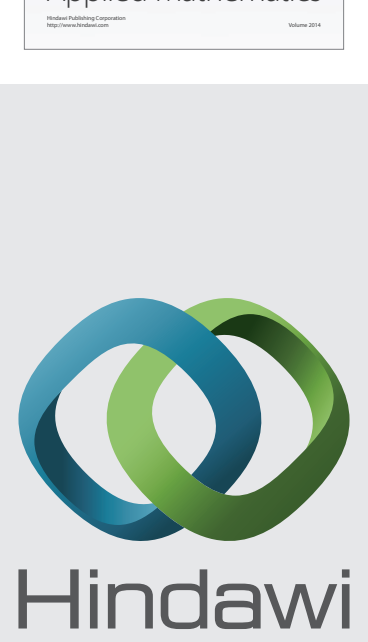

Submit your manuscripts at http://www.hindawi.com
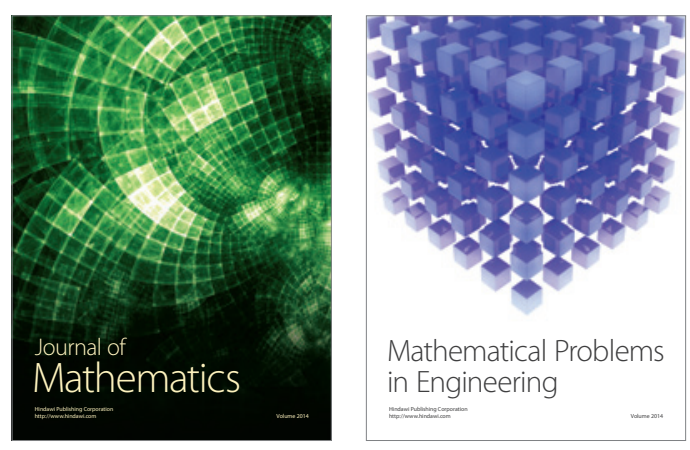

Mathematical Problems in Engineering
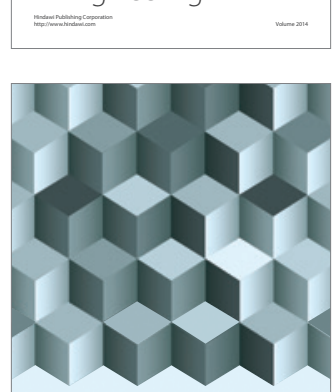

Journal of

Function Spaces
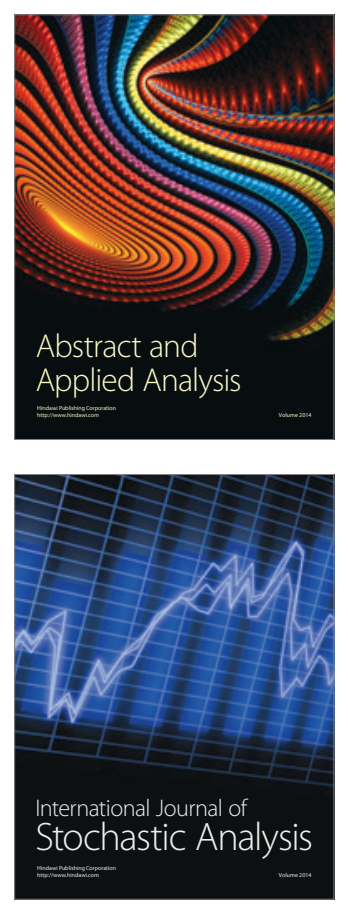

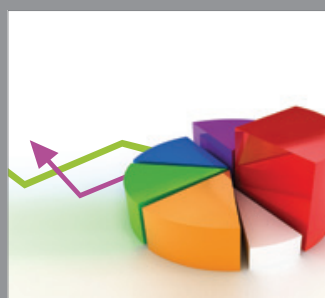

ournal of

Probability and Statistics

Promensencen
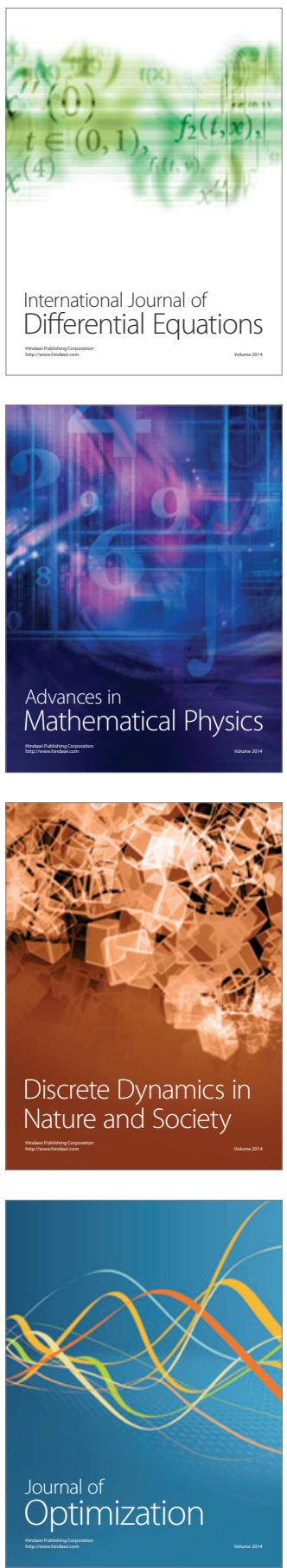